OPEN ACCESS

Edited by:

Kasper Stoy,

IT University of Copenhagen,

Denmark

Reviewed by:

Dimitris Tsakiris,

FORTH Institute of Computer

Science, Greece

Naveen Kuppuswamy,

Toyota Research Institute (TRI),

United States

*Correspondence:

Francesco Corucci

f.corucci@santannapisa.it

Specialty section:

This article was submitted to Bionics and Biomimetics,

a section of the journal

Frontiers in Robotics and $\mathrm{Al}$

Received: 01 February 2017 Accepted: 29 June 2017

Published: 17 July 2017

Citation:

Corucci F, Cheney N, Kriegman S, Bongard J and Laschi C (2017) Evolutionary Developmental Soft Robotics As a Framework to Study Intelligence and Adaptive Behavior

in Animals and Plants.

Front. Robot. Al 4:34.

doi: 10.3389/frobt.2017.00034

\section{Evolutionary Developmental Soft Robotics As a Framework to Study Intelligence and Adaptive Behavior in Animals and Plants}

\author{
Francesco Corucci ${ }^{1,2 *}$, Nick Cheney ${ }^{2,3}$, Sam Kriegman ${ }^{2}$, Josh Bongard ${ }^{2}$ and Cecilia Laschi ${ }^{1}$ \\ ${ }^{1}$ The BioRobotics Institute, Scuola Superiore Sant'Anna, Pisa, Italy, ${ }^{2}$ Morphology, Evolution \& Cognition Laboratory, \\ University of Vermont, Burlington, VT, United States, ${ }^{3}$ Department of Biological Statistics and Computational Biology, Cornell \\ University, Ithaca, NY, United States
}

In this paper, a comprehensive methodology and simulation framework will be reviewed, designed in order to study the emergence of adaptive and intelligent behavior in generic soft-bodied creatures. By incorporating artificial evolutionary and developmental processes, the system allows to evolve complete creatures (brain, body, developmental properties, sensory, control system, etc.) for different task environments. Whether the evolved creatures will resemble animals or plants is in general not known a priori, and depends on the specific task environment set up by the experimenter. In this regard, the system may offer a unique opportunity to explore differences and similarities between these two worlds. Different material properties can be simulated and optimized, from a continuum of soft/stiff materials, to the interconnection of heterogeneous structures, both found in animals and plants alike. The adopted genetic encoding and simulation environment are particularly suitable in order to evolve distributed sensory and control systems, which play a particularly important role in plants. After a general description of the system some case studies will be presented, focusing on the emergent properties of the evolved creatures. Particular emphasis will be on some unifying concepts that are thought to play an important role in the emergence of intelligent and adaptive behavior across both the animal and plant kingdoms, such as morphological computation and morphological developmental plasticity. Overall, with this paper, we hope to draw attention on set of tools, methodologies, ideas and results, which may be relevant to researchers interested in plant-inspired robotics and intelligence.

Keywords: evolutionary robotics, developmental robotics, soft robotics, evo-devo-soro, plant intelligence, animal intelligence, morphological computation, morphological developmental plasticity

\section{INTRODUCTION}

As robotic technology progresses, the main barriers to the creation of truly adaptive and intelligent robotic systems remain mostly conceptual ones. Researchers in biologically inspired robotics (Pfeifer et al., 2007) have typically looked for answers in biology, studying animals (Cacucciolo et al., 2014; Calisti et al., 2014, 2015; Ijspeert, 2014) and, more recently, plants (Pandolfi and Izzo, 2013; Hamann et al., 2015; Temirel et al., 2016; Sadeghi et al., 2017), with the aim of extracting 
general principles underlying the evolutionary success of these creatures, then applying these insights to the development of more effective and efficient robotic systems (Corucci et al., 2015a,b; Giorgio-Serchi et al., 2017). One product of this intellectual procedure is the soft robotics field (Rus and Tolley, 2015; Laschi et al., 2016), arising from the realization that the material characteristics of the body-and softness in particular-play a crucial role in the robust and adaptive behavior of biological and artificial creatures (Pfeifer et al., 2013).

Although bioinspired robot design often results in useful knowledge and new technological solutions, more general tools may be needed in order to gain a deeper understanding and ability to implement general forms of intelligent and adaptive behavior. Particularly, the conditions under which such behavior emerges in soft-bodied creatures-plants, animals, and robots alike-are still largely unknown. This consideration has motivated recent studies on evolutionary developmental soft robotics (evo-devo-soro) (Rieffel et al., 2014; Corucci, 2017), in which a different perspective on bio-inspiration is embraced: instead of taking inspiration from the specific soft animals and plants found in nature today, the natural processes that shaped these creatures (evolution, development) are replicated instead (in silico) (Bongard, 2013), and the emergent properties of artificially evolved creatures analyzed. The idea is that the possibility to manipulate artificial evolutionary and developmental processes, to simulate many different task environments and to access all aspects of evolved creatures (genotype, phenotype, sensory information, etc.) will enable a deeper understanding of the basic phenomena underlying adaptive and intelligent behavior in soft-bodied creatures. These kinds of simulation studies also allow to detach from current technological constraints, developing theories and concepts that can inform neighboring fields. Finally, in addition to supporting hypothesis testing, these techniques effectively constitute powerful design automation tools (Hiller and Lipson, 2009), allowing to devise a wide array of optimized designs for different task environments, that may be soon automatically fabricated (Hiller and Lipson, 2012; MacCurdy et al., 2016; Wehner et al., 2016) and deployed (Lipson and Pollack, 2000; Bongard et al., 2006; Cully et al., 2015).

In this paper, a general evo-devo-soro approach and simulation framework will be described, along with some case studies and results that we consider to be relevant to the study of intelligent and adaptive behavior in soft-bodied creatures, and, as such, to that of plants and plant-inspired systems as well. Able to produce both animal-like (Sect. 3.1) and plant-like creatures (Sect. 3.2), the system exhibits a number of peculiar features (highlighted throughout the paper) which make it particularly promising for application in this latter domain, distinguishing it from alternative approaches (Von Mammen and Jacob, 2009; Zamuda and Brest, 2014; Veenstra et al., 2015, 2016; Wahby et al., 2015, 2016). Particular focus will be on our attempt to integrate biologically plausible developmental dynamics into the system (Sect. 2.4), as well as on phenomena such as morphological computation (Hauser et al., 2014) (Sect. 3.2) and morphological developmental plasticity (Moczek et al., 2011) (Sect. 3.3), which span across both the plant and animal realms.

\section{METHODS AND MATERIALS}

\subsection{Virtual Environment}

Our simulation environment is based on the VoxCAD simulator (Hiller and Lipson, 2014). VoxCAD allows the quantitatively accurate simulation (Hiller and Lipson, 2012) of the static and dynamic behavior of free-form 3D multi-material structures, which can be characterized by large deformations and heterogeneous properties (e.g., density, stiffness): these features make it particularly suitable for simulating plant-like morphologies, which are instead usually approximated by rigid segments interconnected by joints (Veenstra et al., 2016). The simulator, based on a mass-spring lattice of voxels, was considerably extended with custom features to support our experiments, and coupled with a high-level Python library, ${ }^{1}$ were a state of the art evolutionary algorithm (Sect. 2.2) and genetic encoding (Sect. 2.3) have been implemented.

\subsection{Artificial Evolution}

The cornerstone of our bottom-up approach to the study of intelligence and adaptive behavior is evolution, which is here simulated in silico by means of evolutionary algorithms (Bongard, 2013). The process starts from a randomly initialized population of diverse individuals, whose characteristics (phenotypic traits) are encoded in data structures representing their genotype. The genotype can be altered by evolution through genetic operators such as mutation and crossover. The population of simulated robots then evolves over a number of generations: at each generation the current population is evaluated in the task environment at hand. The fitness of each individual is assessed by a function which assigns one scalar number (or more, in multi-objective settings) to each individual. Individuals who perform better with respect to the task at hand get higher chances of surviving to the next generation and reproducing, breeding offspring that consist in randomly modified copies of themselves.

In our framework, a multi-objective evolutionary algorithm was implemented, which allows to optimize robots against several objectives while also promoting population diversity (Schmidt and Lipson, 2011).

\subsection{Genetic Representation/Encoding}

Our genetic representation is based on a very general and evolvable network encoding known as Compositional Pattern Producing Networks (CPPNs) (Stanley, 2007). Designed to capture the formation of regular patterns during development without modeling developmental dynamics per se (Stanley, 2006), CPPNs are networks that convolve input spatial information about a cell to produce outputs (interpreted as phenotypic traits) that tend to exhibit spatial regularities such as symmetry, repetition, and repetition with variation. Both the topology and the connection weights of the CPPN are evolved through a CPPN-NEAT (Stanley, 2007) inspired algorithm. A complete phenotype is achieved by querying the evolved CPPN at every

${ }^{1}$ https://github.com/skriegman/evosoro. 
location of the workspace: as neighboring cells have similar spatial information (coordinates), they also tend to produce similar outputs from the networks, creating a bias toward continuous and spatially organized patches of given material, as found in animals and plants. This representation is also particularly suitable in order to encode distributed properties such the parameters associated with distributed sensory and control systems, very important when evolving artificial plants. Both CPPN inputs and outputs are real-valued, which means that the system automatically supports continuous phenotypes of arbitrarily resolution.

The experimenter needs to decide how to structure the genetic material (inputs, outputs, and number of evolved CPPNs) and how to interpret CPPNs outputs into phenotypic traits. A single CPPN with several outputs can be evolved, promoting the reuse of genotypic information as shared substructures of the same CPPN can contribute to different traits. Nevertheless, monolithic CPPNs are more prone to pleiotropic effects (i.e., mutations on a gene affecting two or more phenotypic traits), which can be detrimental in some cases. An alternative choice is to evolve several independent networks, if genotypic modularity is deemed to be enforced.

\subsection{Development}

Similarly to other encodings [e.g., grammar-based ones, often used to evolve artificial plants (Prusinkiewicz and Lindenmayer, 2012)], CPPNs are designed to produce "regularity without development" (Stanley, 2006), in that they are able to produce regular phenotypic spatial patterns without unfolding a physical process over ontogenetic time. However, there may be other important aspects of development that these approaches may be overlooking.

\subsubsection{The Role of Time in Development}

It has been suggested that changes in merely the timing of developmental events plays a fundamental role in the evolution of morphological innovations (Gould, 1977; McKinney and McNamara, 1991; Hall, 1999). A developmental process may be shifted in ontogenetic time, prolonged or shortened, accelerated or retarded, leading to changes in size and shape: this mechanism is known as heterochrony. There are compelling similarities between juvenile chimpanzees and humans, that are lost due to a different timing of developmental events. Another example is the late suppression of brain developmental processes in humans with respect to that of other primates, which is thought to be partially responsible for the increased brain size (McNamara, 2012).

\subsubsection{The Role of Feedback Loops in Development}

An important consequence arising from the lack of an ontogenetic timescale is that adaptive feedback loops-i.e., control mechanisms which regulate development over the lifetime of an individual-are neglected as well. This may be extremely relevant, as there are several examples from the biological world (Davis, 2014) suggesting that developmental dynamics are not hard-coded in the genotype, but are actually the result of self-organizing, adaptive feedback loops. For example, the skin grows to exactly cover the body, yet its exact dimensions cannot be encoded in the genes, as they can be altered by a number of factors during the lifetime (e.g., diet, physical exercise, diseases, etc.). It has been found that skin cells respond to mechanical tension by proliferating in the direction of the stimulation: as the body grows, tension is applied to the existing skin, which ends up growing in the same directions. As a result of skin growth tension is reduced, which slows down the proliferation of skin cells. Other elegant examples are the adaptive formation of the cytoskeleton within cells, and the growth of the capillary system following oxygen needs (Davis, 2014).

\subsubsection{The Role of Proprioceptive and Exteroceptive Stimuli in Development}

By overlooking the ontogenetic time scale, development does not have a chance to rely on proprioceptive and exteroceptive stimuli experienced over the lifetime, which are known to play an important role in biological development. We have already mentioned how skin growth depends on mechanical tension sensed by the tissue. Similar sensory-dependent adaptations also occur in other types of tissues (Davis, 1867), and in bones as well (Wolff, 1986), which remodel under mechanical loads. Other examples are observable in the plant kingdom, such as the phototropism behavior (growth in response to light), or the growth of roots following gradients of nutrients in the soil.

Whether these aspects are important in the evolution of artificial creatures is an open area of investigation. Nevertheless, their importance in the biological world would suggest that this may actually be the case.

\subsubsection{Time-Dependent Environment-Mediated Morphological Developmental Plasticity}

For the aforementioned reasons, starting from Corucci et al. (2016b) time-dependent environment-mediated developmental processes are modeled within the system, based on distributed sensing and control. The inputs and outputs of these control loops can be selected by evolution itself, along with the quality and magnitude of the developmental adaptation (adaptation rates). In addition to selecting a pool of potential inputs (proprioceptive, exteroceptive) and outputs (control variables), the experimenter selects a general form for the developmental controllers, which so far ensured that development acts at a slower time scale with respect to the usual sensorimotor dynamics. This sharp temporal separation is inspired by the animal world, where there is often a clear distinction between development and control, both in terms of temporal resolution and morphological/neurological effects (e.g., muscle contraction differs from muscle growth). In plants and soft creatures in general this distinction is less sharp (e.g., plants grow and soft creatures deform in order to move), which may open interesting directions of investigation (Sect. 4).

\section{CASE STUDIES}

\subsection{Evolution of Animal-Like Creatures}

When the task environment required locomotion (Cheney et al., 2013; Corucci et al., 2016a), the system was able to evolve a wide array of effective and animal-like morphologies (Figure 1C), 

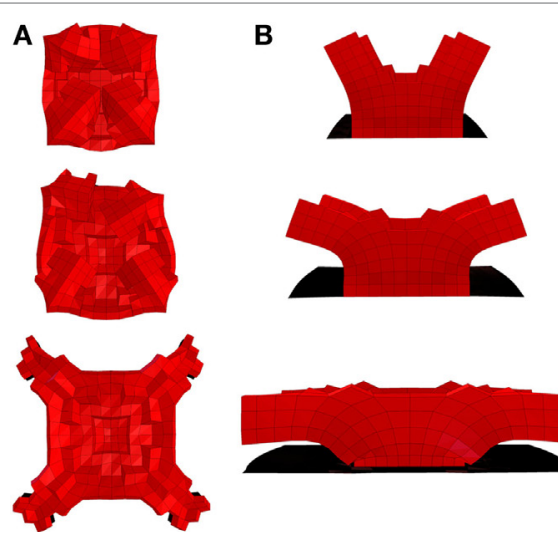

C
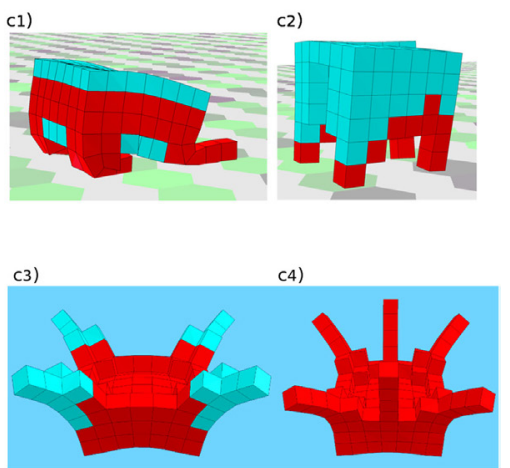

FIGURE 1 | Plant and animal-like creatures evolved by the system for different task environments. (A,B) Two sample plant-like robots, evolved to perform phototropism (see them in action here: https://youtu.be/Cw2SwPNwcfM). Red tissue expands in response to light stimuli. Different growth stages are shown as ontogenetic time unfolds from top to bottom. Both robots exploit passive dynamics and the interaction with the environment (e.g., gravity) in order to perform the task with a remarkably simple growth controller. Branching and symmetry are emergent traits. (A) Top view, four light sources (not depicted) are present at the four corners. The robot expands in response to light stimuli and unfolds under the effect of gravity, resembling a blooming flower. (B) Front view, two lateral light sources (not depicted) are present. A perfect self-sustained cantilevered structure can be noted, where the curvature needed to point toward the lateral light sources is achieved passively. Results of this study suggest that taking into account compliance and material properties is of paramount importance in order to evolve effective plant-like creatures exploiting morphological computation. (C) Four sample animal-like robots evolved to locomote in different environments. Red voxels are volumetrically actuated through a sinusoidal open-loop signal, while light blue ones are passive. (c1,c2) Two quadrupeds evolved to walk on land. As opposed to the more sprawled posture of the soft robot in (c1), a stiffer material (c2) allows an upright position and a proper walking pattern. (c3,c4) Two jellyfish/octopus-like creatures evolved to swim in a watery environment. Video: https://youtu.be/4ZqdvYrZ3ro.

exhibiting diverse locomotion strategies such as swimming, crawling, walking, and running. Results achieved in this setting also suggested how softness can contribute to the evolution of more life-like creatures, as opposed to traditional attempts based on rigid body segments (Sims, 1994).

\subsection{Evolution of Plant-Like Creatures: The Importance of Material Properties and Morphological Computation}

In Corucci et al. (2016b) light sources are placed in the environment, robots are fixed to the ground, and rewarded for phototropism. Developmental adaptation, based on distributed sensing and actuation, entails here volumetric variations in response to sensed light levels. A number of effective plant-like robots have evolved in this setting, characterized by emergent symmetric branching structures (Figures 1A,B). This setting also provided some insights concerning plant-like intelligence. It was found that material properties (i.e., stiffness, here set by the experimenter) have a remarkable impact on evolution's ability to exploit morphological computation (Hauser et al., 2011, 2014) in growing, plant-like, soft creatures. When these were suitable to the task at hand, evolution was able to discover solutions that exploited morphological computation, in that they were characterized by simple growth controllers, sufficient to generate effective behavior thanks to an appropriate morphology and the exploitation of passive dynamics in place of active control. When material properties were not suitable to the task, evolution produced less capable robots, characterized by more complex growth controllers, due to their inability to outsource part of the control to their morphology. This result also indicates that taking into account softness and complex body dynamics is extremely important when evolving artificial plants, as opposed to approaches based on more static, rigid kinematic chains (Veenstra et al., 2016).

\subsection{Evolution of Environment-Mediated Morphological Developmental Plasticity: Adaptivity and Robustness}

Extending Corucci et al. (2016b), we are now further investigating the implications of environment-mediated morphological developmental plasticity, ${ }^{2}$ i.e., the ability of a creature to modify some aspects of its body during its lifetime in response to proprioceptive and exteroceptive information. In Figure 2, an example is reported in which evolving this ability enables robustness to changing environments and the generalization of an evolved behavior to previously unseen conditions. Robots are tasked with locomotion: all voxels are actuated in phase with a fast volumetric actuation, and morphology is evolved along with an initial stiffness distribution. Additionally, taking inspiration from Wolff's law of bones remodeling (Sect. 2.4.3), each voxel can optionally adapt its material stiffness over ontogenetic time, based on the mechanical load (internal pressure and/or stress) experienced over its lifetime. The sensory information selected to drive the developmental change, its quality (stiffen/soften in response to stimulation) and magnitude (adaptation rate) are themselves under evolutionary control. Development is here based on the temporal integration of the proprioceptive stimuli.

In preliminary experiments (Figure 2), the fittest robot evolved a developmental strategy that stiffened parts of the

${ }^{2}$ This is similar to the concept of morphosis (Corucci et al., 2015c; Hauser and Corucci, 2017), although interpreted in a less dynamic sense. 
A a1)

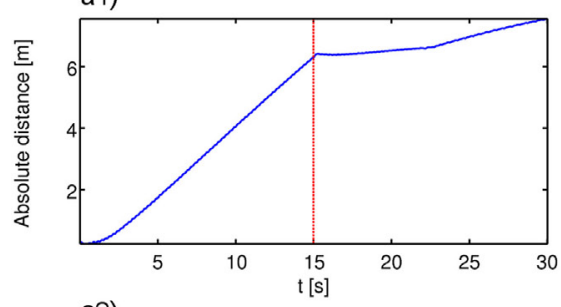

a2)

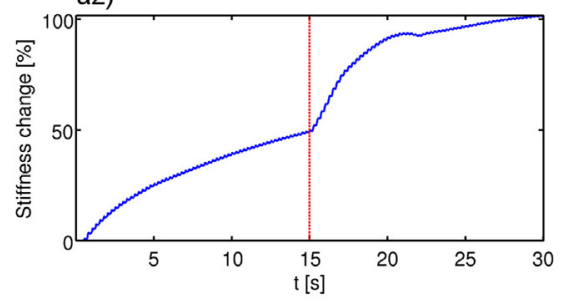

B
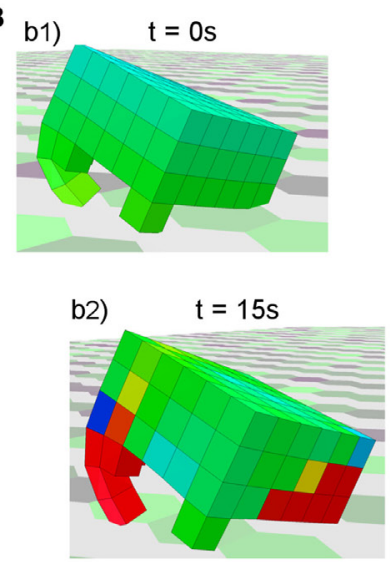
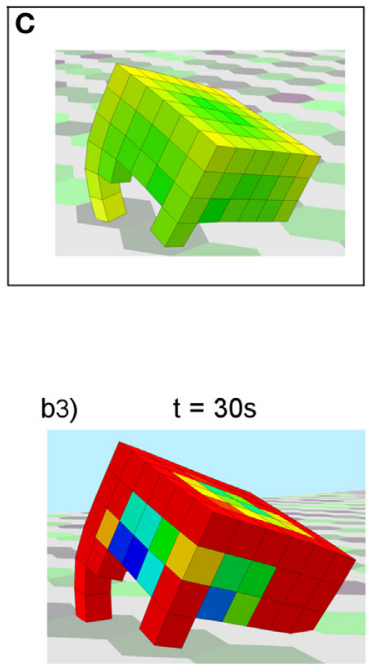

FIGURE 2 | An example of how environment-mediated morphological developmental plasticity can entail increased adaptivity and robustness in dynamic environments. Although demonstrated in a locomotion task, this ability is a constituent element of plant intelligent and adaptive behavior, and it is easy to foresee an application of these concepts to a more plant-inspired domain. (A) (a1) Distance traveled by the robot over time. (a2) Entity of the developmental adaptation (\% stiffness change). (B) A successful robots at three different points in time during its life. Colors represent material stiffness (warmer $\rightarrow$ stiffer), which is under both evolutionary and developmental control. (b1) The robot at its birth ( $t=0 \mathrm{~s}$ ), with its evolved stiffness distribution. (b2) Final form after experiencing a gravity of $g=-9.81 \mathrm{~m} / \mathrm{s}^{2}$ for $15 \mathrm{~s}$. Similarly to what happens in biology, parts of the body that are subject to repeated mechanical stimulation tend to strengthen. Note that the small lateral legs do not touch the ground under the first gravity level. To the best of our knowledge, this is the first example of an artificially evolved Wolff's law for simulated soft-bodied creatures. (b3) Final form after experiencing a new gravity level of $2 \mathrm{~g}$, to which the robot was never exposed during optimization. After a sudden drop in the locomotion performance (see plot (a1), immediately after $t=15 \mathrm{~s}$ ), a new developmental adaptation (a2) allows the robot to start locomoting again even in the new, previously unseen situation, retaining almost $40 \%$ of its original speed. In addition to further stiffening the limbs that are subject to impacts with the ground (note how the shorter lateral limbs touch the ground under the increased gravity level), a stiff "skeletal" structure/frame emerges when gravity is increased, allowing to better sustain and distribute the increased mechanical load. (C) The distribution of the adaptation rates responsible for the developmental change (warmer colors denote higher stiffening in response to mechanical stimulation). See the robot in action here: https://youtu.be/olO2y--mCXE. A close-up of the sensory stimulation driving the adaptive change can be seen here: https://youtu.be/POh359b2iA4.

body more subject to mechanical stimulation during the lifetime (pressure was selected), resulting in a soft back/spine and stiff points of contacts with the ground (Figure 2, b2). ${ }^{3}$ To the best of our knowledge, this is the first example of an artificially evolved Wolff's law for soft robots. After half evaluation time in the same conditions under which it was evolved, the robot is exposed to a doubled gravity acceleration, in order to assess the robustness and generality of the evolved developmental strategy. Predictably, the robot performance drops at first (Figure 2, a1), but the increased mechanical load promptly results in a new developmental adaptation (Figure 2, a2), in which the robot develops even more stiff appendages, as well as a stiff frame/backbone that allows to distribute the increased mechanical load over the morphology (Figure 2, b3). After this adaptive change the robot is able to regain its locomotion ability, reaching almost $40 \%$ of its original speed despite the dramatic environmental change.

This type of evolved mechanisms may contribute to building more adaptive and resilient soft machines in the near future. It is also to be noted that although demonstrated in a locomotion task, the abilities described in this paragraph are at the core of plant intelligent and adaptive behavior.

${ }^{3}$ Given enough time, in a static environment, this developmental change might be eventually genetically assimilated.

\section{DISCUSSION AND CONCLUSION}

In this paper, a general methodology and simulation framework have been reviewed that allow one to test hypotheses related to the evolution of intelligence and adaptive behavior in soft creatures. A common criticism to this type of approach is against its reliance on simulated robots and environments, as opposed to experiments conducted with hardware operating in the real world. While the use of simulation certainly poses some challenges (Jakobi et al., 1995), these are being gradually overcome in the context of rigid robotics (Lipson and Pollack, 2000; Bongard et al., 2006; Cully et al., 2015), and a similar trend may very soon take place in soft robotics as well. Additionally, physical simulation does allow to simulate realistic sensorimotor dynamics. Virtual creatures are embodied: their actions have an effect on the simulated environment, which in turns impacts their sensations. Finally, simulated studies are not meant to replace experiments performed with real hardware, but rather to inform and complement them.

A number of features were described that make the system not only suitable to evolve animal-like creatures, but also plant-like ones. The fact that these different solutions can coexist in the same design space-and be selected or not-could enable interesting studies on the boundaries and constitutive elements of plant and animal intelligence. Our attempts to 
incorporate biologically plausible developmental dynamics into the system have been discussed, which take into account time and environmental stimuli. Some case studies were described in order to suggest the importance of material properties and morphological computation in modeling and evolving growing plant-like creatures, which support our choice of dealing with soft, multi-material creatures instead of rigid ones. The potential benefits in terms of adaptivity arising from evolved mechanisms of environment-mediated morphological developmental plasticity were pointed out as well: although demonstrated in a locomotion task, the latter ability is at the core of plant intelligent and adaptive behavior.

The generality of the system is challenging many of our preconceived assumptions on the evolution, development, and movement of soft animals and plants. What is the difference between morphological development and movement, from the perspective of a soft-bodied creature? Under which conditions will an evolved creature be born with some innate specialized morphological structures, as opposed to evolving general purpose ones, or developing them during its lifetime? While these questions remain open for the time being, we hope to stimulate further discussion in fields concerned with plant, animal, and robot intelligence, as well as to draw attention on a set of tools and techniques that we regard as promising in order to investigate these fascinating questions.

\section{REFERENCES}

Bongard, J., Zykov, V., and Lipson, H. (2006). Resilient machines through continuous self-modeling. Science 314, 1118-1121. doi:10.1126/science.1133687

Bongard, J. C. (2013). Evolutionary robotics. Commun. ACM 56, 74-83. doi:10.1145/2492007.2493883

Cacucciolo, V., Corucci, F., Cianchetti, M., and Laschi, C. (2014). "Evolving optimal swimming in different fluids: a study inspired by batoid fishes," in Biomimetic and Biohybrid Systems, eds A. Duff, N. F. Lepora, A. Mura, T. J. Prescott, and P. F. M. J. Verschure (Cham: Springer International Publishing), 23-34.

Calisti, M., Corucci, F., Arienti, A., and Laschi, C. (2014). "Bipedal walking of an octopus-inspired robot," in Biomimetic and Biohybrid Systems, eds A. Duff, N. F. Lepora, A. Mura, T. J. Prescott, and P. F. M. J. Verschure (Cham: Springer), $35-46$.

Calisti, M., Corucci, F., Arienti, A., and Laschi, C. (2015). Dynamics of underwater legged locomotion: modeling and experiments on an octopus-inspired robot. Bioinspir. Biomim. 10, 046012. doi:10.1088/1748-3190/10/4/046012

Cheney, N., MacCurdy, R., Clune, J., and Lipson, H. (2013). "Unshackling evolution: evolving soft robots with multiple materials and a powerful generative encoding," in Proceedings of the 15th Annual Conference on Genetic and Evolutionary Computation (Amsterdam: ACM), 167-174.

Corucci, F. (2017). "Evolutionary developmental soft robotics: towards adaptive and intelligent soft machines following nature's approach to design," in Soft Robotics: Trends, Applications and Challenges (Cham: Springer International Publishing), 111-116.

Corucci, F., Calisti, M., Hauser, H., and Laschi, C. (2015a). "Evolutionary discovery of self-stabilized dynamic gaits for a soft underwater legged robot," in Proceedings of the 17th International Conference on Advanced Robotics (ICAR2015) (Istanbul: IEEE), 337-344.

Corucci, F., Calisti, M., Hauser, H., and Laschi, C. (2015b). "Novelty-based evolutionary design of morphing underwater robots," in Proceedings of the 2015 Genetic and Evolutionary Computation Conference (GECCO2015) (Madrid: ACM), 145-152.

Corucci, F., Calisti, M., Hauser, H., and Laschi, C. (2015c). "Shaping the body to shape the behavior: a more active role of the morphology in the brain-body trade-off," in Proceedings of the 13th European Conference on Artificial Life (ECAL2015), Late Breaking Proceedings, New York, 7-8.

\section{AUTHOR CONTRIBUTIONS}

All listed authors have made a substantial, direct, and intellectual contribution to the work and have approved it for publication. Particularly, FC designed and conducted the experiments, data collection, and data analysis, while FC, NC, SK, CL, and JB contributed to designing this research, interpreting results, and editing the manuscript.

\section{ACKNOWLEDGMENTS}

This work was supported by the Vermont Advanced Computing Core.

\section{FUNDING}

This work was supported by the RoboCom++ project (funded by the European Union, FLAG-ERA JTC2016), the National Science Foundation awards INSPIRE-1344227, PECASE-0953837, and the United States Army Research Office award W911NF-16-1-0304. FC is supported by grant agreement no. 604102 (Human Brain Project) funded by the European Union Seventh Framework Programme (FP7/2007-2013). NC is supported by NASA Space Technology Research Fellowship \#NNX13AL37H.

Corucci, F., Cheney, N., Lipson, H., Laschi, C., and Bongard, J. (2016a). "Evolving swimming soft-bodied creatures," in ALIFE XV, The Fifteenth International Conference on the Synthesis and Simulation of Living Systems, Late Breaking Proceedings, Cancún, 6.

Corucci, F., Cheney, N., Lipson, H., Laschi, C., and Bongard, J. (2016b). "Material properties affect evolution's ability to exploit morphological computation in growing soft-bodied creatures," in ALIFE XV, The Fifteenth International Conference on the Synthesis and Simulation of Living Systems (Cancún: MIT Press), 234-241.

Cully, A., Clune, J., Tarapore, D., and Mouret, J.-B. (2015). Robots that can adapt like animals. Nature 521, 503-507. doi:10.1038/nature14422

Davis, H. (1867). Conservative Surgery. New York: D. Appleton \& Company.

Davis, J. (2014). A Closed Loop. Available at: https://aeon.co/essays/the-feedbackloop-is-a-better-symbol-of-life-than-the-helix

Giorgio-Serchi, F., Arienti, A., Corucci, F., Giorelli, M., and Laschi, C. (2017). Hybrid parameter identification of a multi-modal underwater soft robot. Bioinspir. Biomim. 12, 025007. doi:10.1088/1748-3190/aa5ccc

Gould, S. J. (1977). Ontogeny and Phylogeny. Cambridge, MA: Harvard University Press.

Hall, B. K. (1999). Evolutionary Developmental Biology. Berlin: Springer Science \& Business Media.

Hamann, H., Wahby, M., Schmickl, T., Zahadat, P., Hofstadler, D., Stoy, K., et al. (2015). "Flora robotica-mixed societies of symbiotic robot-plant bio-hybrids," in Computational Intelligence, 2015 IEEE Symposium Series On (Cape Town: IEEE), 1102-1109.

Hauser, H., and Corucci, F. (2017). "Morphosis taking morphological computation to the next level," in Soft Robotics: Trends, Applications and Challenges (Cham: Springer International Publishing), 117-122.

Hauser, H., Füchslin, R. M., and Pfeifer, R. (2014). Opinions and Outlooks on Morphological Computation. Zürich. Available at: http://www.merlin.uzh.ch/ publication/show/10528

Hauser, H., Ijspeert, A. J., Füchslin, R. M., Pfeifer, R., and Maass, W. (2011). Towards a theoretical foundation for morphological computation with compliant bodies. Biol. Cybern. 105, 355-370. doi:10.1007/s00422-012-0471-0

Hiller, J., and Lipson, H. (2012). Automatic design and manufacture of soft robots. IEEE Trans. Robot. 28, 457-466. doi:10.1109/TRO.2011.2172702 
Hiller, J., and Lipson, H. (2014). Dynamic simulation of soft multimaterial 3d-printed objects. Soft Robot. 1, 88-101. doi:10.1089/soro.2013.0010

Hiller, J. D., and Lipson, H. (2009). "Multi material topological optimization of structures and mechanisms," in Proceedings of the 11th Annual Conference on Genetic and Evolutionary Computation (Montreal, QC: ACM), 1521-1528.

Ijspeert, A. J. (2014). Biorobotics: using robots to emulate and investigate agile locomotion. Science 346, 196-203. doi:10.1126/science.1254486

Jakobi, N., Husbands, P., and Harvey, I. (1995). Noise and the reality gap: the use of simulation in evolutionary robotics. Adv. Artif. Life 929, 704-720. doi:10.1007/3-540-59496-5_337

Laschi, C., Mazzolai, B., and Cianchetti, M. (2016). Soft robotics: technologies and systems pushing the boundaries of robot abilities. Sci. Robot. 1, eaah3690. doi:10.1126/scirobotics.aah3690

Lipson, H., and Pollack, J. B. (2000). Automatic design and manufacture of robotic lifeforms. Nature 406, 974-978. doi:10.1038/35023115

MacCurdy, R., Katzschmann, R., Kim, Y., and Rus, D. (2016). "Printable hydraulics: a method for fabricating robots by $3 \mathrm{~d}$ co-printing solids and liquids," in Robotics and Automation (ICRA), 2016 IEEE International Conference On (Stockholm: IEEE), 3878-3885.

McKinney, M. L., and McNamara, K. J. (1991). "Heterochrony," in Heterochrony (New York: Springer), 1-12.

McNamara, K. J. (2012). Heterochrony: the evolution of development. Evol. Educ. Outreach 5, 203. doi:10.1007/s12052-012-0420-3

Moczek, A. P., Sultan, S., Foster, S., Ledón-Rettig, C., Dworkin, I., Nijhout, H. F., et al. (2011). The role of developmental plasticity in evolutionary innovation. Proc. Royal Soc. London B Biol. Sci. 278, 2705-2713. doi:10.1098/rspb.2011. 0971

Pandolfi, C., and Izzo, D. (2013). Biomimetics on seed dispersal: survey and insights for space exploration. Bioinspir. Biomim. 8, 025003. doi:10.1088/1748-3182/ $8 / 2 / 025003$

Pfeifer, R., Lungarella, M., and Iida, F. (2007). Self-organization, embodiment, and biologically inspired robotics. Science 318, 1088-1093. doi:10.1126/ science. 1145803

Pfeifer, R., Marques, H. G., and Iida, F. (2013). "Soft robotics: the next generation of intelligent machines," in Proceedings of the Twenty-Third international joint conference on Artificial Intelligence (Beijing: AAAI Press), 5-11.

Prusinkiewicz, P., and Lindenmayer, A. (2012). The Algorithmic Beauty of Plants. New York: Springer Science \& Business Media.

Rieffel, J., Knox, D., Smith, S., and Trimmer, B. (2014). Growing and evolving soft robots. Artif. Life 20, 143-162. doi:10.1162/ARTL_a_00101

Rus, D., and Tolley, M. T. (2015). Design, fabrication and control of soft robots. Nature 521, 467-475. doi:10.1038/nature14543

Sadeghi, A., Mondini, A., Dottore, E. D., Mattoli, V., Beccai, L., Taccola, S., et al. (2017). A plant-inspired robot with soft differential bending capabilities. Bioinspir. Biomim. 12, 015001. doi:10.1088/1748-3190/12/1/ 015001

Schmidt, M., and Lipson, H. (2011). "Age-fitness pareto optimization," in Genetic Programming Theory and Practice VIII (New York: Springer), 129-146.
Sims, K. (1994). "Evolving virtual creatures," in Proceedings of the 21st Annual Conference on Computer Graphics and Interactive Techniques (Orlando, FL: ACM), 15-22.

Stanley, K. O. (2006). "Exploiting regularity without development," in Proceedings of the AAAI Fall Symposium on Developmental Systems (Menlo Park, CA: AAAI Press), 37.

Stanley, K. O. (2007). Compositional pattern producing networks: a novel abstraction of development. Genet. Program. Evol. Mach. 8, 131-162. doi:10.1007/ s10710-007-9028-8

Temirel, M., Yenilmez, B., Knowlton, S., Walker, J., Joshi, A., and Tasoglu, S. (2016). Three-dimensional-printed carnivorous plant with snap trap. D Print. Addit. Manuf. 3, 244-251. doi:10.1089/3dp.2016.0036

Veenstra, F., Faina, A., Støy, K., and Risi, S. (2015). "Evolving self-organizing simulated plant-inspired robots," in Workshop on Methods for Self-Organizing Distributed Systems, Laubusch.

Veenstra, F., Faina, A., Stoy, K., and Risi, S. (2016). "Generating artificial plant morphologies for function and aesthetics through evolving L-systems," in Proceedings of the Artificial Life Conference 2016 (Cancùn: MIT Press).

Von Mammen, S., and Jacob, C. (2009). The evolution of swarm grammars-growing trees, crafting art, and bottom-up design. IEEE Comput. Intell. Mag. 4, 10-19.

Wahby, M., Hofstadler, D. N., Heinrich, M. K., Zahadat, P., and Hamann, H. (2016). "An evolutionary robotics approach to the control of plant growth and motion: modeling plants and crossing the reality gap," in Self-Adaptive and Self-Organizing Systems (SASO), 2016 IEEE 10th International Conference On (Augsburg: IEEE), 21-30.

Wahby, M., Soorati, M. D., von Mammen, S., and Hamann, H. (2015). "Evolution of controllers for robot-plant bio-hybrids: a simple case study using a model of plant growth and motion," in Proc. of the 25th Workshop on Computational Intelligence (Dortmund: KIT Scientific Publishing), 67-86.

Wehner,M., Truby, R.L.,Fitzgerald,D.J.,Mosadegh, B., Whitesides, G.M.,Lewis,J. A., et al. (2016). An integrated design and fabrication strategy for entirely soft, autonomous robots. Nature 536, 451-455. doi:10.1038/nature19100

Wolff, J. (1986). The Law of Bone Remodelling. Berlin: Springer Science \& Business Media.

Zamuda, A., and Brest, J. (2014). Vectorized procedural models for animated trees reconstruction using differential evolution. Inf. Sci. 278, 1-21. doi:10.1016/j. ins.2014.04.037

Conflict of Interest Statement: The authors declare that the research was conducted in the absence of any commercial or financial relationships that could be construed as a potential conflict of interest.

Copyright (c) 2017 Corucci, Cheney, Kriegman, Bongard and Laschi. This is an open-access article distributed under the terms of the Creative Commons Attribution License (CC BY). The use, distribution or reproduction in other forums is permitted, provided the original author(s) or licensor are credited and that the original publication in this journal is cited, in accordance with accepted academic practice. No use, distribution or reproduction is permitted which does not comply with these terms. 